\title{
Antisynthetase Syndrome associated with mycobacterium tuberculosis infection, presenting as an acute respiratory failure
}

\begin{abstract}
Antisynthetase syndrome (ASS) is characterized by myositis, interstitial lung disease, Raynaud's phenomenon, fever and mechanics hands. Diagnosis is confirmed with the detection of an antibody directed against anti-aminoacyl-transfer-RNA synthetases (ARS). The most common anti-ARS antibody is anti-Jo-1. Opportunistic infections are common causes of mortality in patients with autoimmune diseases. Immunosuppressive treatment further contributes to the risk of infection.

We report a rare case of a 68 year-old man diagnosed with antisynthetase syndrome associated to a pulmonary tuberculosis infection, revealed with an acute respiratory failure. The diagnosis of this rare combination of a connective tissue disease and tuberculosis revealed with an acute respiratory failure is difficult in a previously asymptomatic patient. Early diagnosis and immunosuppressive therapy associated to antituberculosis treatment started precociously prevented the disease progression and resulted in a good outcome.
\end{abstract}

Keywords: anti-synthetase syndrome, respiratory failure, interstitial lung disease, tuberculosis, mycobacterium tuberculosis
Volume 8 Issue I - 202I

\author{
Amani Ben Mansour, ${ }^{1,4}$ Soumaya Ben Saad, ${ }^{1,4}$ \\ Sadok Yaalaoui, ${ }^{2,4}$ Hedia Bellali, ${ }^{3,4}$ Hafaoua \\ Daghfous, ${ }^{1,4}$ Fatma Tritar ${ }^{1,4}$ \\ 'Pneumology "C" Department,Abderrahman Mami Hospital, \\ Tunisia \\ ${ }^{2}$ Department of Immunology,Abderrahman Mami Hospital, \\ Tunisia \\ ${ }^{3}$ Research Laboratory "Analysis of the Effects of Environmental \\ and Climate Change on Health", Department of Epidemiology \\ and Statistics, Abderrahmen Mami Hospital, Tunisia \\ ${ }^{4}$ Faculty of Medicine of Tunis, Tunis El Manar University, Tunis, \\ Tunisia
}

Correspondence: Amani Ben Mansour, Pneumology " $\mathrm{C}$ " Department, Mami Ariana Hospital, Medical faculty of Tunis, Tunisia,Tel +21652737428; Email benmansour_aman@yahoo.fr

\section{Introduction}

Idiopathic inflammatory myopathies are rare autoimmune diseases characterized by proximal muscle weakness in association with various clinical involvements of the joints, skin, lungs, and esophagus. ${ }^{1}$ One subset, antisynthetase syndrome, requires an antibody directed against aminoacyl transfer RNA. Pulmonary tuberculosis in autoimmune conditions is may be accredited to various immune irregularities as well as to treatment with immunosuppressive therapy. Herein, we report a 68-year-old man who presents with fever and acute respiratory failure, who was diagnosed with anti-JO1 antibody associated antisynthetase syndrome. Associated pulmonary tuberculosis was secondary identified.

\section{Case presentation}

A 68 year-old man, builder, with non-smoking history was admitted to the pulmonology department for acute dyspnea, weakness and fever, with rapid deterioration of respiratory conditions. He has a history of erysipelas of the lower limb 10 years ago, single kidney (organ donation for his daughter in 2018) and newly diagnosed diabetes. He did not report any other symptoms and had been in good health until the last 4 weeks. The physical examination revealed the following: body temperature $37.8^{\circ} \mathrm{C}$, respiratory rate $24 \mathrm{breaths} /$ minute, blood pressure $150 / 75 \mathrm{mmHg}$, pulse rate $107 \mathrm{beats} /$ minute, and oxygen saturation $81 \%$ on room air. Fine crackles were heard at the base of the lungs. A rough appearance of the hands was noted (Figure 1). Skin exam was negative for rash. There was no lymphadenopathy. The rest of the exam was normal.

At admission, the patient had acute respiratory failure. Arterial blood gas analysis with oxygen $8 \mathrm{~L} / \mathrm{min}$ showed a $\mathrm{PaO} 2$ of 67 $\mathrm{mmHg}, \mathrm{PaCO}$ of $31 \mathrm{mmHg}$, pH of 7.47 , and $\mathrm{HCO}^{3}$ of $23 \mathrm{mEq} / \mathrm{L}$. A thoracic radiograph showed multiple pulmonary infiltrates consistent with interstitial lung disease (Figure 2). Echocardiogram showed normal left ventricular function. Laboratory investigations revealed neutrophilic leukocytosis (white blood cells 14200/UL, neutrophils $11640 / \mathrm{mL}$, lymphocytes $1670 / \mathrm{mL}$ ); elevated sedimentation rate $136(<20 \mathrm{~mm} / \mathrm{h})$; elevated lactated shydrogenase (LDH),313 $\mathrm{U} / \mathrm{L}$; creatine phosphokinase (CPK) level,24 U/L (0-195 U/L) aspartate aminotransferase (AST) level, $15 \mathrm{U} / \mathrm{L}$ (6-34 U/L); alanine transaminase (ALT) level, 16 (6-34 U/L); and C-reactive protein, 44 $\mathrm{mg} / \mathrm{dL}(0-5 \mathrm{mg} / \mathrm{dL})$. HIV test was negative.

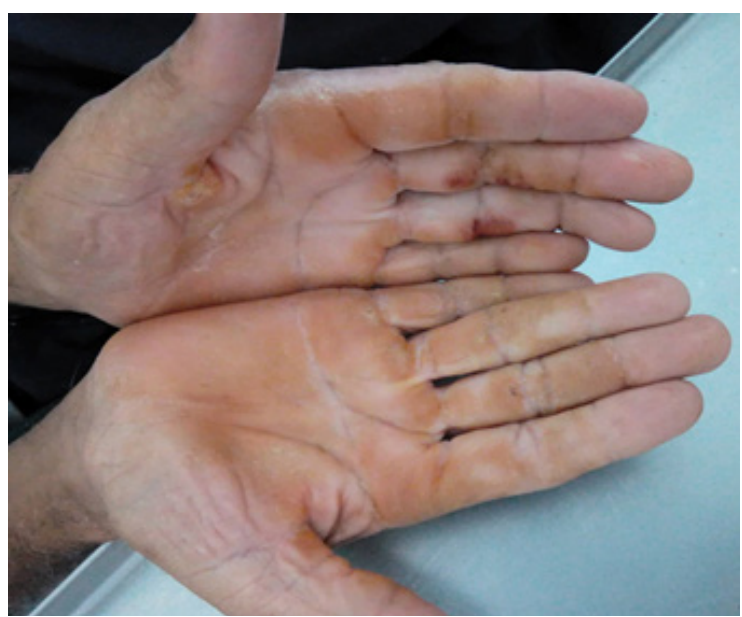

Figure I Fissural and erythematous hyperkeratosis of the palmar face of the hands (mechanic's hands).

He was diagnosed with severe community-acquired pneumonia and treated with oxygen, intravenous corticosteroids and antibiotics (amoxicillin-clavulanic acid and Erythromycin).

High-resolution computed tomography of the chest showed bilateral micronodular opacities, thickening of septal lines, tractionbronchiectasis, all consistent with bilateral interstitial non-specific pneumonia (Figure 3). 


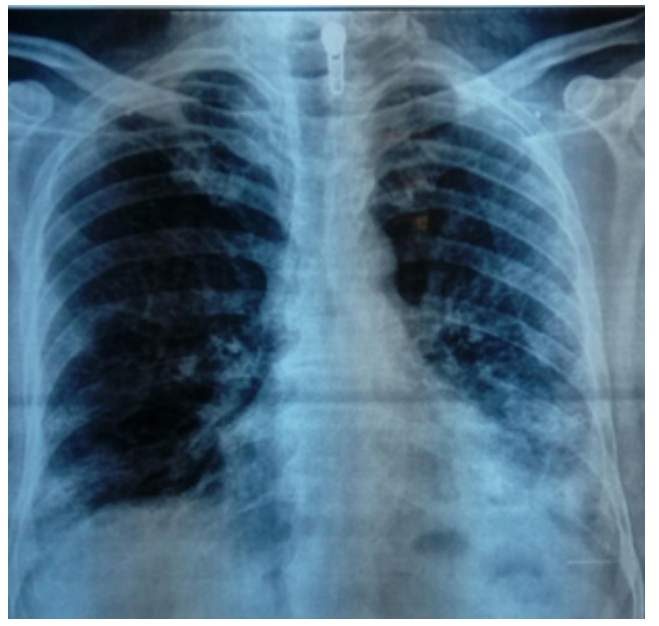

Figure 2 Multiple pulmonary infiltrates consistent with interstitial lung disease.

On the third day of hospitalization, the patient's general and respiratory conditions worsened. Atypical bacterial pathogens serologies was negative as well as RT-PCR influenza A (H1N1), negative cytobacteriological examination of sputum and negative mycobacterium tuberculosis sputum.

Since there was no evidence of bacterial, fungal, or viral infection, we suspected inflammatory myopathy with interstitial lung disease.

Thus, we checked specific markers for connective tissue diseases. Laboratory immunological tests revealed increased anti-nuclear antigen antibodies, as well as positive anti-extractable nuclear antigen (anti-Jo-1 antibodies positive and anti-SSA-Ro52 positive); rheumatoid factor and anti-neutrophil cytoplasmic antibodies $(\mathrm{C}-$ ANCA and P-ANCA) were at normal values. Bronchoalveolar lavage was not performed initially. The diagnosis of Antisynthetase syndrom was made, and the patient continued prednisone at the dose of $60 \mathrm{mg} /$ day $(1 \mathrm{mg} / \mathrm{kg})$. Cyclophosphamide pulse therapy $(750 \mathrm{mg}$. once every 45 days $\times 6$ ) was programmed.

In the workup before beginning immunosuppressive treatment, a thoraco-abdomino-pelvic tomography was performed. In addition to interstitial lung disease lesions, mediastinal and hilar adenomegaly, lingular excavated lesion and confluent centrilobular micronodules of the left lung was noted (Figure 4). Rapid multiplex-PCR for Mycobacterium tuberculosis diagnosis using sputum samples was positive. The diagnosis of associated pulmonary tuberculosis was retained.
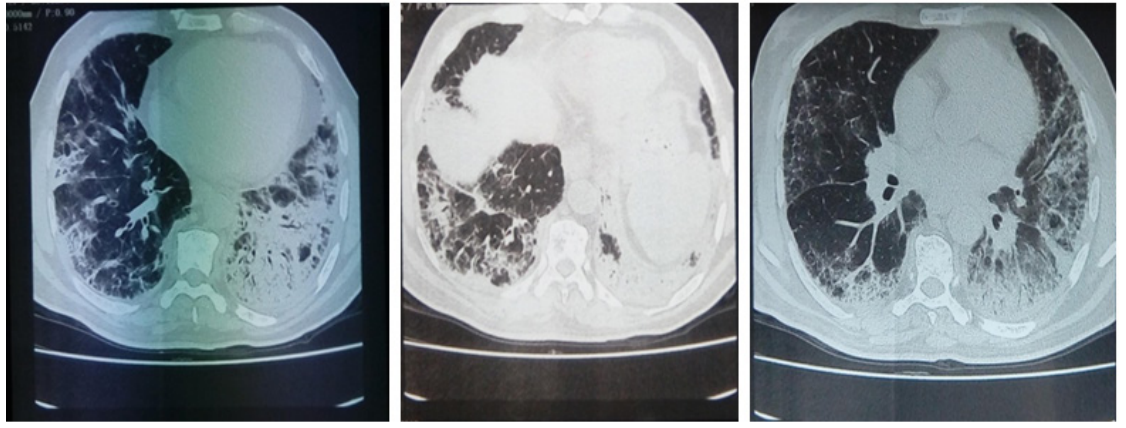

Figure 3 Chest High-resolution computed tomography showing bilateral micronodular opacities, thickening of septal lines, traction-bronchiectasis, all consistent with bilateral interstitial nonspecific pneumonia.
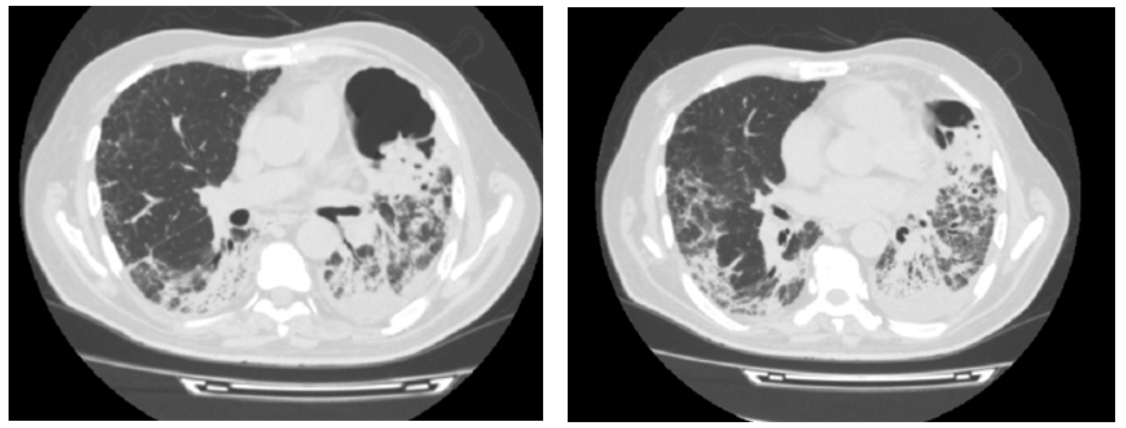

Figure 4 Chest High-resolution computed tomography showing lingular excavated lesion and confluent centrilobular micronodules of the left lung.

In addition to corticoids treatments, anti-tuberculosis drug has been started (Isoniazid $300 \mathrm{mg} / \mathrm{j}$, Rifampicin $600 \mathrm{mg} / \mathrm{j}$, Pyrazinamid $1600 \mathrm{mg} / \mathrm{j}$, Ethambutol $1100 \mathrm{mg} / \mathrm{j})$.

Three weeks after the begining of anti-tuberculosis treatment, the respiratory effort had improved, and the patient was discharged. At short-term follow-up, he reported significant improvement in his dyspnea. Patient's respiratory condition improved $(\mathrm{PaO} 282.9 \mathrm{mmHg}$, PaCO $33.5 \mathrm{mmHg}$, pH 7.46, and HCO32 $24 \mathrm{mEq} / \mathrm{L}$ on 3 1/mn oxygen
); and laboratory values for blood cell count, CPK, LDH, and CRP returned to normal ranges within three weeks.

\section{Discussion}

The diagnosis of polymyositis/ dermatomyositis PM/DMrelated to interstitial lung disease (ILD) is not difficult in patients with established disease or in newly diagnosed patients with typical disease manifestations. ${ }^{2}$ However, PM/DM may not be suspected 
to be the cause of ILD when ILD is the only manifestation. ${ }^{3}$ Severe respiratory failure as the presenting feature of ILD associated with ASS is extremely rare. Acute respiratory failure is an extremely rare presentation of the ASS syndrome. ${ }^{2}$ Clinical suspicion of polymyositis is high where muscle pain or tenderness is obvious, but these symptoms are present only in $50 \%$ of the cases. ${ }^{4}$ Antibodies are directed against aminoacyl transfer RNA (tRNA) synthetases. Eight antibodies have been discovered, including anti-Jo-1 (most common), anti-EJ, antiPL7, anti-PL12, anti-OJ, anti-KS, anti-ZO, and anti-Ha. ${ }^{5}$ Nearly all are associated with ILD, but myositis is closely associated with AntiJo-1, Anti-PL-7, and Anti-EJ. ${ }^{6}$ Patients with non-Jo-1 antibodies are shown to have lower survival rates. ${ }^{7}$ Corticosteroids remain the cornerstone of initial empiric treatment for inflammatory myopathy. ${ }^{8}$ Among patients with antisynthetase syndrome-related ILD, the response to therapy with prednisone is heterogeneous, with $30-40 \%$ of the subjects showing improvement and $20-40 \%$ being stabilized. ${ }^{9}, 10$ Other immunosuppressive drugs should be considered at the outset of treatment, particularly in ASS and other severe and progressive manifestations of ILD. ${ }^{11}$ For patients who have responded poorly to the conventional pulse steroid therapy, increasing the intensity of pulse cyclophosphamide, cyclosporine, or other immunosuppressive therapy earlier remain the best approach. ${ }^{12}$

Individuals with systemic connective tissue diseases are at increased risk for developing infection, either from immune abnormalities, from the disease itself, or from immunosuppressive treatment. Bacterial infections are most common, but viral and fungal infections also contributed to increased morbidity and mortality. ${ }^{13}$

A study of eighteen patients with PM/DM showed a high frequency of opportunistic infections, with more than $50 \%$ being fungal. ${ }^{14}$ The incidence rate of M.tuberculosis infection in idiopathic inflammatory myopathies is greater than the incidence in the general population. ${ }^{15}$ Extrapulmonary patterns of tuberculosis have also been described to be more common in IIMs; in a retrospective case series of thirty patients who had tuberculosis and systemic rheumatic disease, $2 / 3$ of the patients had extrapulmonary tuberculosis while only $1 / 3$ had pulmonary tuberculosis. ${ }^{16}$ It is possible that the immunosuppression in our patient led to re-activation of tuberculosis.

\section{Conclusion}

This case illustrates an extremely rare association in the medical literature between anti-synthetase syndrome and mycobacterium tuberculosis infection. Early diagnosis and appropriate treatment lead to better prognosis.

\section{Conflicts of interest}

There are no conflicts of interest.

\section{Acknowledgement}

None.

\section{References}

1. Lundberg IE, de Visser M, Werth VP. Classification of myositis. Nat Rev Rheumatol. 2018;14(15):269-278.

2. Piroddi IM, Ferraioli G, Barlascini C, et al. Severe respiratory failure as a presenting feature of an interstitial lung disease associated with antisynthetase syndrome (ASS). Respir Investig. 2016;54(4):284-288.

3. Yousem SA, Gibson K, Kaminski N, et al. The pulmonary histopathologic manifestations of the antiJo-1 tRNA synthetase syndrome. Mod Pathol. 2010;23(6):1199-1204.

4. Walton J. Disorders of voluntary muscle. In: Weatherall DJ, et al., editors. The Oxford Textbook of Medicine. $3^{\text {rd }}$ edn. Oxford: Oxford University Press 1996; ID:415664158.

5. Witt LJ, Curran JJ, Strek ME. The Diagnosis and Treatment of Antisynthetase Syndrome. Clin Pulm Med. 2016;23:218-226.

6. Hamaguchi Y, Fujimoto M, Matsushita T, et al. Common and distinct clinical features in adult patients with antiaminoacyl-tRNA synthetase antibodies: heterogeneity within the syndrome. PLos One. 2013;8(4):e60442.

7. Aggarwal R, Cassidy E, Fertig N, et al. Patients with non-Jo-1 antitRNAsynthetase autoantibodies have worse survival than $\mathrm{J}_{0}-1$ positive patients. Ann Rheum Dis. 2013;73(1):227-232.

8. Oddis CV. Idiopathic inflammatory myopathy: management and prognosis. Rheum Dis Clin North Am. 2002;28(4):979-1001.

9. Frazier AR, Miller RD. Interstitial pneumonitis in association with polymyositis and dermatomyositis. Chest. 1974;65(4):403-407.

10. Salmeron G, Greenberg SD, Lidsky MD. Polymyositis and diffuse interstitial lung disease. A review of the pulmonary histopathologic findings. Arch Intern Med. 1981;141(8):1005-1010.

11. Saketkoo LA, Ascherman DP, Cottin V, et al. Interstitial Lung Disease in Idiopathic Inflammatory Myopathy. Curr Rheumatol Rev. 2010;6(2):108-119.

12. Kim SH, Park IN. Acute Respiratory Distress Syndrome as the Initial Clinical Manifestation of an Antisynthetase Syndrome. Tuberc Respir Dis (Seoul). 2016;79(3):188-192.

13. Alarcón GS Infections in systemic connective tissue diseases: systemic lupus erythematosus, scleroderma, and polymyositis/dermatomyositis. Infect Dis Clin North Am. 2006;20(4):849-875

14. Marie I, Hachulla E, Chérin P, et al. Opportunistic infections in polymyositis and dermatomyositis. Arthritis Rheum. 2005;53(2):155165.

15. Kim HA, Yoo CD, Baek HJ, et al. Mycobacterium tuberculosis infection in a corticosteroid-treated rheumatic disease patient population. Clin Exp Rheumatol. 1998;16(1):9-13.

16. Hernández-Cruz B, Sifuentes-Osornio J, Ponce-de-León Rosales $\mathrm{S}$, et al. Mycobacterium tuberculosis infection in patients with systemic rheumatic diseases. A case-series. Clin Exp Rheumaton. 1999;17(3):289-296. 